\title{
THE EFFECT OF ENERGY MINIMIZATION ON THE MOLECULAR DOCKING OF ACETONE-BASED OXINDOLE DERIVATIVES
}

\begin{abstract}
Yesaya Reformyada Nusantoro and Arif Fadlan ${ }^{*}$
Department of Chemistry, Faculty of Science and Data Analytics, Institut Teknologi Sepuluh Nopember

Jl. Teknik Mesin No.175, Keputih, Sukolilo, Surabaya, East Java 60115, Indonesia

* correspondence, tel/fax: 031-5943353/5928314, email: afadlan@chem.its.ac.id

\section{ABSTRACT}

In silico study by molecular docking, drug discovery, and virtual screening are useful for obtaining compounds with promising biological activity. The force fields energy minimization in molecular docking is the overall process to produce better geometry estimation and ligandreceptor affinity. In this study, the divide and conquer algorithm based on the Mikowski matrix in MarvinSketch and the conjugate gradient algorithm of Open Babel were used to minimise acetone-based oxindole derivatives in indoleamine 2,3-dioxygenase 1 (IDO1). The results showed that the binding energy produced by MarvinSketch was generally better than the binding energy obtained with Open Babel. The visualization of molecular docking results indicated that the poses and hydrogen bonding, halogen bonding and $\pi-\pi$ interactions are different between MarvinSketch, Open Babel, and no energy minimization. The results revealed that energy minimization affects the molecular docking results.
\end{abstract}

Keywords: Molecular Docking; Energy Minimization; MMFF94; Oxindole, IDO1

\section{INTRODUCTION}

Molecular docking is a simulation of a protein (enzyme) and small molecules (ligands) interactions by the computational procedure. Molecular docking predicts the geometry and behaviour of ligands in the binding sites of target enzymes. Thus, this technique identifies ligands' correct orientations when bound to a protein and forms a stable complex $[1,2]$. The biological function and biochemical processes of the protein may be enhanced or inhibited by the interactions of the ligands in the supra- molecular complex formed [3]. Based on the binding site of a protein, docking can be categorized as selective and blind dockings. The docking process can be performed as flexible ligand docking, rigid body docking, and flexible docking depend on the flexibility of the ligands and protein.

The docking procedure consists of two main steps; sampling and scoring. The first step involves the generation and prediction of conformations of the ligands and their orientation in the protein's active site (known as pose). The scoring function as the second step evaluates the best pose and ranks the 
ligands based on the binding affinity. The docking process should predict the best pose and the affinity of the ligands accurately $[3,4]$. The structure of ligands is usually optimized by energy minimization to achieve a conformation with the lowest energy that illustrates their stability. This optimization step is essential since the two-dimensional structure of the ligands, as drawn, are not energetically stable. The minimization is stopping when the local energy minimum is reached as the energy minimization, and the program is operated. However, this point is not representing the most stable conformer of the ligands. A global energy minimum depicting the most stable conformer can be obtained using suitable algorithms [5]. The force fields with the Steepest Descent algorithm are usually used for this purpose. The force field evaluates the atomic interactions, including van der Waals and electrostatic interactions, bond-stretching, bending, and torsion forces. The force field is determined based on experimental data and by the mechanical calculations based on the laws of physics [6].

In the present study, the energy minimization of 5,7-dichloro-3-hydroxy-3-(2oxopropyl)-2,3-dihydro-1H-indol-2-one

(Figure 1) was studied by using the divide and conquer algorithm based on the Mikowski matrix in MarvinSketch and with the conjugate gradient algorithm of Open Babel in indoleamine 2,3-dioxygenase 1 (IDO1). The results were compared to 1-methyl-Ltryptophan (L-1MT), a standard inhibitor of IDO1. This research is expected to provide information about the effect of energy minimization in the molecular docking process.<smiles>CC(=O)CC1(O)C(=O)Nc2c(Cl)cc(Cl)cc21</smiles>

1<smiles>CN1C(=O)C(C[C@H](N)C(=O)O)c2ccccc21</smiles>

L-1MT
Figure 1. Structure of $\mathbf{1}$ and L-1MT

\section{METHODS}

This study was performed according to previously reported method [7]. Docking was started by IDO1 enzyme preparation and compound 1 and L-1MT optimization. The docking was then performed on 2D0T macromolecule followed by visualization. The steps of this study are described as follow:

\section{Macromolecule preparation}

The crystal structure of indoleamine 2,3-dioxygenase 1 (PDB ID: 2DOT) with a resolution of $2.30 \AA$ was chosen for this study [8]. PyMOL [9] was used for removing water molecules and 4-phenyl imidazole (PIM) cocrystallized ligand followed by the addition of hydrogen atoms.

\section{Ligand preparation and optimization}

The two- and three-dimensional (2D/3D) structure of the ligands and their protonation steps were developed using MarvinSketch [9]. The MMFF94 energy minimization of the ligands was carried out by divide and conquer algorithm based on the Mikowski matrix in MarvinSketch [9] and by conjugate gradient algorithm in Open Babel [10] of PyRx [11] (200 steps and a minimum root mean square (RMS) gradient of 0.0001 $\mathrm{kcal} / \mathrm{mol} / \mathrm{A})$. 


\section{Molecular docking}

Molecular docking was accomplished using AutoDock Vina [12] in PyRx [11]. The grid of the receptor was set in the area around the active site of the protein with sizes $X 12$ $\AA, Y 12 \AA, Z 12 \AA$ and dimensions X $59.9 \AA$, $Y 53.1 \AA$, Z $18.8 \AA$.

\section{RESULTS AND DISCUSSION}

\section{Macromolecule preparation}

The 2D0T macromolecule at a resolution of $2.30 \AA$ was retrieved from a worldwide protein data bank (wwPDB). This macromolecule consists of two identical chains, e.g. a large domain with $15 \alpha$-helical chains and a small domain with $9 \alpha$-helical chains and two $\beta$ chains [7]. The preparation of 2D0T was started by removing water molecules and extracting PIM co-crystallized ligand. It was reported that removing water molecules could increase the accuracy of docking by finding the most representative binding pose of ligand and protein [13]. The extraction of PIM cocrystallized ligand aimed to provide the binding site, which was defined at a coordinate of $X=$ $59.9 \AA, Y=53.0 \AA$, and $Z=18.8 \AA$.

\section{Ligand preparation and optimization}

The two dimensional (2D) structure of ligand $\mathbf{1}$ with the addition of all hydrogen atoms was prepared using MarvinSketch. The three dimensional (3D) structure of the ligands was then achieved using the same program by generating coordinates from a temporary coordinate data set that meets the interatomic distance requirements according to the Minkowski approach [14]. The ligands optimization was performed by Merck molecular force field (MMFF94) energy minimization using MarvinSketch (a) and Open Babel (b) and compared to no minimization (c). The energy minimization process produces a conformational structure with the lowest total potential energy [5]. The minimization step of ligand $\mathbf{1}$ provided $\mathbf{1 a}, \mathbf{1} \mathbf{b}$, and 1c conformations with the energy of $32.31,35.72$, and $51.74 \mathrm{kcal} / \mathrm{mol}$, respectively. By portraying the minimization energy pattern of ligand 1 , i.e. $1 a<\mathbf{1 b}<\mathbf{1 c}$, it is recognized that energy minimization affects the stability of ligand 1 . The ligand $1 \mathrm{a}$ produced by MarvinSketch was the most stable conformation among others, $\mathbf{1 b}$ and $\mathbf{1 c}$, as indicated by the smallest energy. This fact indicated the Steepest Descent algorithm of MMFF94 energy minimization fitted with the divide-and-conquer algorithm of MarvinSketch in the characterization of bonds, angles, atomic properties, and dihedral angels of ligand $1[15,16]$. These results also revealed that the divide-and-conquer algorithm works better than the conjugate gradient algorithm of Open Babel. It is well known that the divideand-conquer algorithm solves the problem by dividing it into several sub-problems that are similar to the actual problem but in a smaller size. The algorithm then resolves the subproblems recursively and then combines them to form a solution to the real problem [17]. Meanwhile, the Open Babel energy minimization based on conjugate gradient algorithm breaks the problem using an iterative method for linear equations in the form of $A x=b$ with $a$ matrix $A$ having symmetric positive definitive. In general, this algorithm clarifies a large system of linear equations and looks for the minimum point of 
a quadratic function of a vector so that the search process is expected to be faster [18].

\section{Molecular docking}

Table 1 . The binding affinity of ligands 1 and L-1MT on 2D0T

\begin{tabular}{ccc}
\hline Ligands & Pose & $\begin{array}{c}\text { Binding affinity } \\
\text { (kcal/mol) }\end{array}$ \\
\hline \multirow{2}{*}{ 1a } & 1 & $-1,5$ \\
& 2 & $-0,7$ \\
1b & 3 & 0,9 \\
& 1 & 0,3 \\
1C & 2 & 0,4 \\
& 3 & 0,8 \\
& 1 & $-1,0$ \\
L-1MTa & 2 & $-0,8$ \\
& 3 & $-0,7$ \\
& 1 & -2.3 \\
& 2 & -2.0 \\
& 3 & -0.9 \\
& 5 & -0.8 \\
& 5 & -0.3 \\
L-1MTb & 7 & -0.1 \\
& 1 & 0.1 \\
& 2 & -1.0 \\
& 3 & -0.9 \\
& 4 & -0.6 \\
& 5 & -0.6 \\
& 6 & -0.3 \\
& 7 & 1.5 \\
& 1 & 1.8 \\
& 2 & -1.2 \\
& 3 & -1.0 \\
& 4 & -0.9 \\
& 5 & -0.8 \\
& 6 & 0.0 \\
& 5 & 0.5 \\
& 5 & 1.2 \\
\hline
\end{tabular}

Molecular docking was performed at the binding site of PIM co-crystallized ligand at a coordinate of $X=59.9 \AA, Y=53.1 \AA$, and $Z$ $=18.6 \AA$ within the radius of $12 \AA$. The redocking procedure carried out the validation of the docking process. The results showed that the native ligand was successfully docked back onto its binding site. The alignment of the best pose of docked PIM with co-crystallized PIM ligand produced root-mean-square deviation (RMSD) values of $0.088 \AA$. Visualization of the docked PIM indicated interaction with ferrous ion $\left(\mathrm{Fe}^{2+}\right)$ of $\mathrm{HEM}$ group as in the co-crystallized ligand. The interaction occurs via a metal coordination bond between the nitrogen atom at the 2position of the original ligand and the PIM anchored with theFe ${ }^{2+}$ of the HEM group within a distance of $2.13 \AA$ [3] and $2.60 \AA$, respectively. Based on these results, the redocking procedure is acceptable and can be used for the next evaluation.

Flexible ligand docking where the ligand is flexible and macromolecule are rigid were then evaluated using AutodockVina in Pyrx. Binding affinity determines the strength of ligand-receptor interaction. The more negative binding affinity, the stronger ligand-receptor interaction and the better molecular docking prediction [7]. As shown in Table 1, the binding affinity values from the best pose (pose 1) of each ligand $\mathbf{1 a}, \mathbf{1} \mathbf{b}$, and $\mathbf{1 c}$ were -1.5, 0.3, and $-1.0 \mathrm{kcal} / \mathrm{mol}$. Evaluation of this profile was then taken by looking at L-1MT for comparison. A similar pattern was coincidentally found for L-1MT. Minimization using MarvinSketch yielded the lowest binding affinity value (L$1 \mathrm{MTa}-2.3 \mathrm{kcal} / \mathrm{mol}$ ) L-1MTc and L-1MTb with the value of $-1.2 \mathrm{kcal} / \mathrm{mol}$ and $-1.0 \mathrm{kcal} / \mathrm{mol}$, respectively. Based on the binding affinity, thus the strength order of ligan-receptor interactions was $1 \mathrm{a}>1 \mathrm{c}>1 \mathrm{~b}$ and L-1MTa $>$ L-1MTC > L-1MTb. Both ligand 1 and L-1MT showed a similar profile. These results showed that the strength of ligand-receptor interactions was also influenced by energy minimization. The ligand 1a resulted from minimization using MarvinSketch gave the lowest binding affinity and this result was linearly correlated with the value of minimization energy. In the minimization process, the MMFF94 force field adjusted the 
structures of ligands and yielded a different conformation from the initial before minimization. These conformations were then accommodated in the binding site, and the strength of the interactions was represented based on binding affinity. It is reported that binding affinity is also influenced by the energy of desolvation, conformation, interactions, and motions $[19,20]$.

\section{Interaction analysis}

The docking results were then visualized to determine the interactions and binding mode of the ligands-protein complex. These results were shown in Figure 2 and Table 2. Analysis of binding mode revealed that ligand 1 interacted with Serine-167 residue and HEM group and the side chain of 2D0T. Ligand 1a with the lowest binding affinity is bound to Serin-167 residue through the 5-chloro group $(2.95 \AA)$ by halogenbonding interaction. Interactions through halogen-bonding were also occurred between the 7-chloro group with the nitrogen atom of the HEM pyrrole ring (3.17 $\AA$ ) and with another nitrogen atom from another HEM pyrrole ring
(2.99 Å). Other interactions included halogenbonding through 5-chloro group with Tyrosine126 residue, $\pi-\pi$ stacking interactions through benzene ring with Phenylalanine- 163 residue and HEM group, also hydrophobic interactions with Tyrosine-126 and Phenylalanine-163 residues.

The binding mode analysis of ligand 1 was then compared to L-1MT. This ligand showed a similar binding affinity profile as ligand 1 (Table 2). In general, L-1MT is bound to 2D0T via HEM group, Serine-167 and Glycine-262 residues (Figure 3). L-1MTa showed hydrogen-bonding interaction through nitrogen atom on indole side chain with Serine-167 (2.76 ̊). This ligand is also bound to 2D0T by hydrogen-bonding interactions through oxygen and hydrogen atoms of the carboxyl group with Glycine-262 residue (2.86 and $2.43 \AA$ A). Other hydrogen-bonding interactions also existed through the amino group with Cysteine-129 residue. The analysis also revealed that this ligand formed $\pi-\pi$ stacking interactions through benzene rings with HEM and hydrophobic interactions with Cysteine-129 residue.

Table 2. Interaction of ligands $\mathbf{1}$ and L-1MT to 2D0T macromolecule

\begin{tabular}{|c|c|c|c|c|}
\hline \multirow{2}{*}{ Ligands } & \multicolumn{4}{|c|}{ Interacting residues } \\
\hline & Hydrogen bonds & Halogen bonds & $\pi-\pi$ stacking & Hydrophobic \\
\hline $1 \mathrm{a}$ & 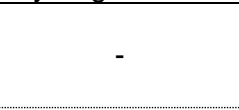 & $\begin{array}{c}\text { HEM } \\
\text { Tyr-126 } \\
\text { Ser-167 }\end{array}$ & $\begin{array}{c}\text { HEM } \\
\text { Phe-163 }\end{array}$ & $\begin{array}{l}\text { Tyr-126 } \\
\text { Phe-163 }\end{array}$ \\
\hline 1b & - & $\begin{array}{c}\text { HEM } \\
\text { Ser-167 } \\
\text { Ser-263 }\end{array}$ & HEM & - \\
\hline 1c & $\begin{array}{l}\text { Gly-262 } \\
\text { Ser-263 }\end{array}$ & $\begin{array}{l}\text { Tyr-126 } \\
\text { Ser-167 } \\
\text { Ser-263 }\end{array}$ & $\begin{array}{c}\text { HEM } \\
\text { Tyr-126 }\end{array}$ & Tyr-126 \\
\hline L-1MTa & $\begin{array}{l}\text { Cys-129 } \\
\text { Ser-167 } \\
\text { Gly-262 }\end{array}$ & - & HEM & Cys-129 \\
\hline L-1MTb & $\begin{array}{l}\text { Cys-129 } \\
\text { Ser-167 } \\
\text { Gly-262 }\end{array}$ & - & $\begin{array}{c}\text { HEM } \\
\text { Tyr-126 }\end{array}$ & $\begin{array}{l}\text { Tyr-126 } \\
\text { Cys-129 }\end{array}$ \\
\hline L-1MTc & $\begin{array}{l}\text { Ser-167 } \\
\text { Gly-262 }\end{array}$ & - & $\begin{array}{c}\text { HEM } \\
\text { Tyr-126 }\end{array}$ & Tyr-126 \\
\hline
\end{tabular}


The explanation mentioned above revealed that energy minimization affects the binding affinity and ligand-receptor interactions. The optimization of ligand $\mathbf{1}$ using MarvinSketch MMFF94 energy minimization produced the most stable ligand 1a with the smallest energy. MarvinSketch's algorithm supported the MMFF94 Steepest Descend algorithm [12-14]. Next, the ligands 1 a and L1MTa generated the lowest binding affinity value than others. The energy minimization affected ligand-receptor interactions [16,17]. Moreover, the different energy minimization was also influenced ligand-protein interaction.
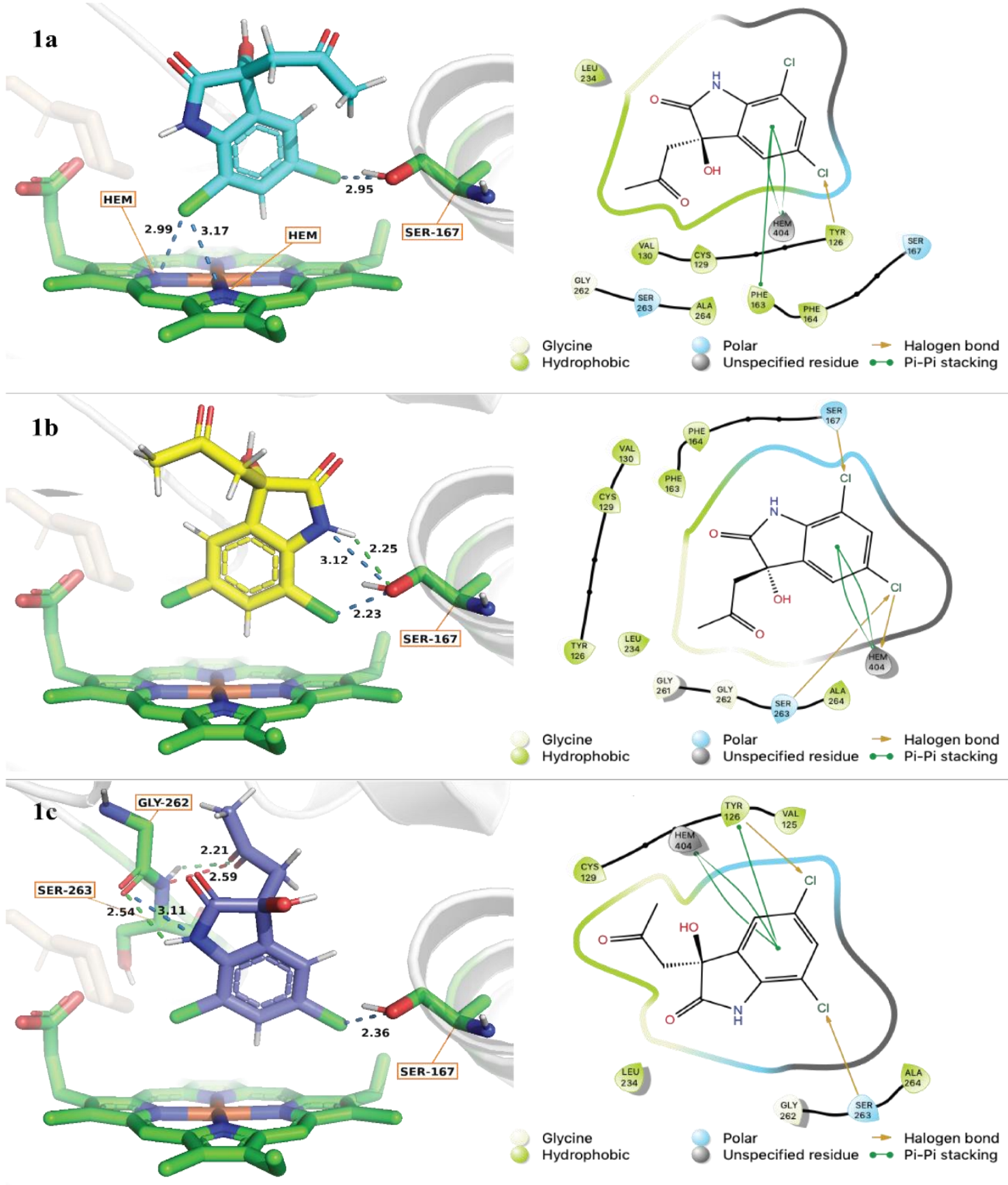

Figure 2. Interactions and binding mode of ligand 1 on 2D0T 


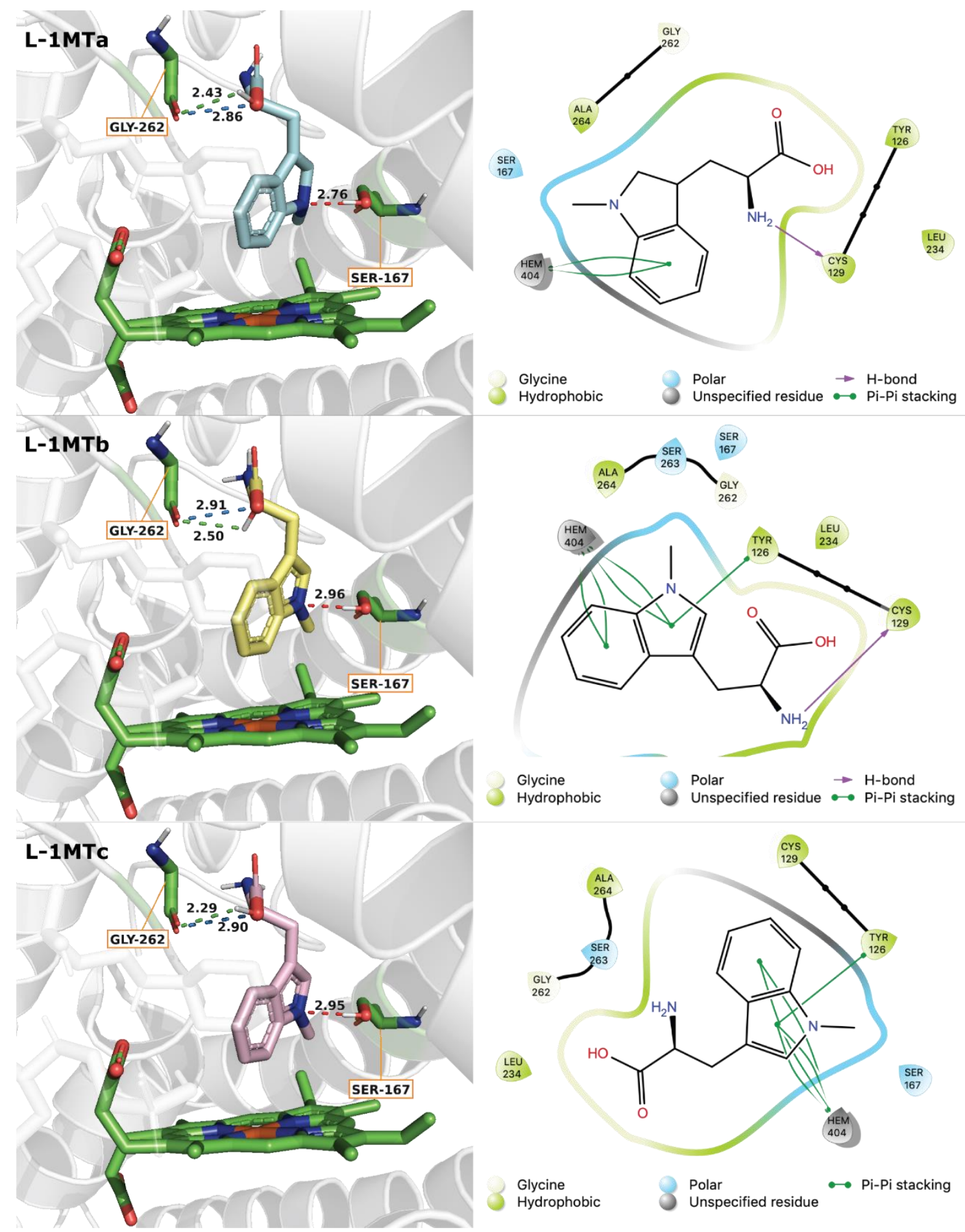

Figure 3. Interactions and binding mode of ligand L-1MT on 2D0T

\section{CONCLUSION}

The MMFF94 is the prevalent energy minimization function in molecular docking. We studied the effect of energy minimization using MMFF94 by MarvinSketch and Open
Babel, and without minimization, of ligand 1 and L-1MT. The RMSD value and interactions analysis indicated that the redocking procedure was valid. This study revealed that minimization affects the total 
potential energy, binding affinity, and ligandreceptor interactions. MarvinSketch for energy minimization provided the best results.

\section{ACKNOWLEDGEMENT}

Authors thank Institut Teknologi Sepuluh Nopember through Penelitian Doktor Baru (852/PKS/ITS/2020).

\section{REFERENCES}

[1] E. March-Vila, L. Pinzi, N. Sturm, A. Tinivella, O. Engkvist, H. Chen, \& G. Rastelli, "On the integration of in silico drug design methods for drug repurposing." Front. Pharmacol., vol. 8 (MAY), pp. 1-7. 2017.

DOI: 10.3389/fphar.2017.00298

[2] E. H. B. Maia, L. C. Assis, T. A. de Oliveira, A. M. da Silva, \& A. G. Taranto, "Structure-based virtual screening: From classical to artificial intelligence." Front. Pharmacol., vol. 8 (April). 2020.

DOI: 10.3389/fchem.2020.00343

[3] K. Roy, S. Kar, \& R. N. Das, "Other related techniques" in Understanding the basics of QSAR for applications in pharmaceutical sciences and risk assessment," K. Roy, S. Kar, R. N. Das (eds), 2015a, pp. 357-425.

DOI:10.1016/B978-0-12-801505-

6.00010-7

[4] S. Perez \& I. Tvaroska, "Carbohydrateprotein interactions: Molecular modelling insights" in advances in carbohydrate Chemistry and Biochemistry, Horton, D. (Ed). vol. 71, 2014, pp: 9-136.

DOI: 10.1016/B978-0-12-800128-

8.00001-7

[5] K. Roy, S. Kar, \& R. N. Das, "Computational chemistry," in Understanding The Basics of QSAR for Applications in Pharmaceutical Sciences Risk Assessment, K. Roy, S.
Kar and R. N. Das (eds.), Elsevier, San Diego, 2015b, pp. 357-425.

DOI:10.1016/B978-0-12-8015056.00005-3

[6] N. L. Allinger, "Conformational analysis. 130. MM2. A hydrocarbon force field utilizing $\mathrm{V} 1$ and $\mathrm{V} 2$ torsional terms," J. Am. Chem. Soc., vol. 99, no. 25, pp. 8127-8134, 1977.

DOI: $10.1021 / \mathrm{ja} 00467 \mathrm{a} 001$

[7] T. Daggupati, K. N. Chitrala, R. Pamanji, \& S. Yeguvapalli, "Molecular screening and analysis of novel therapeutic inhibitors against $\mathrm{c}$-jun $\mathrm{N}$ terminal kinase," Med. Chem. Res., vol. 26, pp. 2112-2118, 2017.

DOI: 10.1007/s00044-017-1919-5

[8] H. Sugimoto, S. Oda, S.-i. Otsuki, T. Hino, T. Yoshida, \& Y. Shiro, "Crystal structure of human indoleamine 2,3dioxygenase: Catalytic mechanism of $\mathrm{O}_{2}$ incorporation by a heme-containing dioxygenase," Proc. Natl. Acade. Sci., vol. 103, no. 8, pp. 2611-2616, 2006. DOI: 10.1073/pnas.0508996103

[9] N. Novichikhina, I. Ilin, A. Taschilova, A. Sulimov, D. Kutov, I. Ledenyova, M. Krysin, K. Shikhaliev, A. Gantseva, E. Gantseva, N. Podoploleva, \& V. Sulimov, "Synthesis, docking, and in vitro anticoagulant activity assay of hybrid derivatives of pyrrolo[3,2,1ij]quinolin-2(1H)-one as new inhibitors of factor Xa and factor Xla," Molecules, vol. 25, pp. 1889, 2020.

DOI: 10.3390/molecules25081889

[10] N. M. O'Boyle, M. Banck, C. A. James, C. Morley, T. Vandermeersch, \& G. R. Hutchison, "Open Babel: An open chemical toolbox" J. Cheminformatics, vol. 3, no. 33, pp. 1-14, 2011. DOI: 10.1186/1758-2946-3-33

[11] S. Dallakyan \& A. J. Olson, "Smallmolecule library screening by docking with PyRx," in Chemical Biology: Methods in Molecular Biology, J. E. Hempel, C. H. Williams, C. C. Hong (Eds.), Springer, New York, 2015. DOI: 10.1007/978-1-4939-2269-7_19 
[12] O. Trott \& A. Olson, "AutoDock Vina: improving the speed and accuracy of docking with a new scoring function, efficient optimization, and multithreading," J. Comput. Chem., vol. 31, no. 2, pp. 455-461, 2009.

DOI: $10.1002 /$ jcc. 21334

[13] D. B. Kitchen, H. Decomez, J. R. Furr, \& J. Bajorath, "Docking and scoring in virtual screening for drug discovery: Methods and applications," Nat. Rev. Drug Discov., vol. 3, no. 11, pp. 935949, 2004.

DOI: $10.1038 / \mathrm{nrd} 1549$

[14] G. Imre, G. Veress, A. Volford, \& O. Farkas, "Molecules from the Minkowski space: An approach to building 3D molecular structures," $J$. Mol. Struct., vol. 666-667, pp. 51-59, 2003.

DOI: 10.1016/j.theochem.2003.08.013

[15] D. Gentile, V. Patamia, A. Scala, M. T. Sciortino, A. Piperno, \& A. Rescifina, "Putative inhibitors of SATS-CoV-2 main protease from a library of marine natural products: A virtual screening and molecular modeling study," Mar. Drugs, vol. 18, no. 4, 225, 2020.

DOI: $10.3390 / \mathrm{md} 18040225$
[16] R. Prihartiningtyas, R. R. Syahdi, M. Y. Putra, \& A. Yanuar, "Establishment of a 3D-structure database for chemical compounds in Indonesian sponges," Pharmacogn. J., vol. 11, no. 6, pp. 1211-1218, 2019.

DOI: $10.5530 /$ pj.2019.11.188

[17] T. Cormen C. Leiserson, R. Rivest, C. Stein, Introduction to Algorithms, The MIT Press, Massachusetts, 2009.

ISBN: 9780262533058

[18] M. Hestenes \& E. Stiefel, "Methods of conjugate gradients for solving linear systems," J. Res. Nat. Bur. Standa., vol. 49, no. 6, pp. 409-436, 1952.

[19] Ajay \& M. Murcko, "Computational methods to predict binding free energy in ligand-receptor complexes," J. Med. Chem., vol. 38, no. 26, pp. 4953-4967, 1995.

DOI: 10.1021/jm00026a001

[20] T. Siebenmorgen \& M. Zacharias, "Computational prediction of proteinprotein binding affinities," WIREs Comput. Mol. Sci., vol. 10, no. e1448, pp. 1-18, 2020.

DOI: $10.1002 /$ wcms. 1448 